Angiogenesis is a complex multistep process by which new capillary structures arise from pre-existing vessels in response to angiogenic stimuli. This process plays a key role during tumorigenesis because the vascular network within the tumor enables malignant cells to establish distant metastases. Thus, it is not surprising that targeting tumors with angiogenesis-based therapy remains a significant area of preclinical and clinical studies. One of the most prominent factors considered as a promising target in such therapy is the Notch ligand Delta-like 4 (DLL4). Emerging evidence suggests that blockade of DLL4 in tumors results in excessive but non-productive angiogenesis which affects tumor growth, even in tumors which are insensitive to anti-VEGF therapy. Nevertheless, the careful evaluation of adverse effects on normal organs' physiology in relation to therapeutic doses of DLL4 inhibitors will be critical for advancement of DLL4 blocking agents in clinical practice.

Key words: Notch signaling, DLL4, angiogenesis, cancer therapy, tumor vasculature.

\section{The Notch ligand Delta-like 4 (DLL4) as a target in angiogenesis-based cancer therapy?}

\author{
Marlena Brzozowa, Romuald Wojnicz, Grażyna Kowalczyk-Ziomek, \\ Krzysztof Helewski
}

Chair and Department of Histology and Embryology, Faculty of Medicine and Dentistry in Zabrze, Medical University of Silesia, Katowice, Poland

\section{Introduction}

Angiogenesis is a complex multistep process by which new capillary structures arise from pre-existing vessels in response to angiogenic stimuli. Although the process of new blood vessel formation occurs primarily during fetal development, it is also detected in adults, in whom both physiological (e.g. wound healing, female menstrual cycle) and pathological (e.g. age-related macular degeneration, solid tumor growth) neovascularization occurs [1]. It has been widely reported that formation of blood vessels appeared as a key process driving tumorigenesis because the vascular network within the tumor enables malignant cells to escape from the primary site and establish distant metastases elsewhere [2]. Therefore, targeting tumors with angiogenesis-based therapy remains a very significant area of preclinical and clinical studies [3, 4]. The main strategies for such therapy have been developed to prevent stimulation of endothelial cells by a rich variety of pro angiogenic molecules released from the extracellular matrix by proteases or secreted both by tumor and stromal cells $[2,5]$. Of all the pro angiogenic molecules, the most significant and well described is vascular endothelial growth factor (VEGF), which is produced at high levels by tumor cells and stromal cells $[6,8]$. It is worth noting that high expression of VEGF is reported as an independent factor predicting poor prognosis in different types of malignant tumors including colon cancer, gastric cancer, prostate cancer, non-small cell lung cancer and cervical cancer [6, 7]. In the context of angiogenesis regulation, the role of VEGF receptors (VEGFR) should also be mentioned. For example, expression of VEGFR2 is detected in the polarized extension of tip cell filopodia, which enables migration along the chemoattractant gradient. The next receptor, VEGFR1, plays a dual role: a negative role during vasculogenesis probably by trapping VEGF-A, and a positive role in postnatal life by a tyrosine kinase-dependent manner [9-11].

As revealed by the studies, activation of the Notch signaling pathway affects multiple aspects of vascular development $[12,13]$. One of the components of the Notch signaling pathway, Delta-like ligand 4 (DLL4) has recently appeared as a critical regulator of tumor angiogenesis and thus as a promising therapeutic target. Emerging evidence suggests that blockade of DLL4 in tumors results in excessive but non-productive angiogenesis, which affects tumor growth, even in tumors which are insensitive to anti-VEGF therapy [1, 14].

In this paper, we discuss the role of DLL4 as a regulator of tumor angiogenesis and promising target in angiogenesis-based cancer therapy.

\section{Notch signaling pathway}

The Notch signaling pathway is an evolutionarily conserved cell signaling system which plays a significant role in cell fate determination and differentiation during embryonal and postnatal development [15] (Fig. 1). In mammalian cells, 
four Notch receptors (Notch1-4) can bind five different ligands: Jag1, Jag2, Delta-like 1, -3, -4. Each of them is synthesized as a precursor protein and within the Golgi apparatus is cleaved at site $\mathrm{S} 1$ by furin-like convertases to generate two subunits. It is important that the extracellular domain of the Notch receptor contains numerous EGF-like repeats. Ligand binding to EGF-like repeats unfolds the Negative Regulatory Region (NRR) permitting cleavage by ADAM metalloproteases at site S2. During the next step, $\boldsymbol{\gamma}$-secretases execute an intramembrane cleavage at site S3, releasing the Notch intracellular domain (NotchIC), which translocates to the nucleus. The Mastermind-like family of proteins (MAML1/2/3) has been demonstrated to be needed for Notch signaling. MAML forms a ternary complex with CBF1-NotchIC and then acts as a transcriptional activator. Following Notch activation two families of bHLH proteins are induced: the hairy/ enhancer-of-split family (HES) and the hairy-related transcription factor family (HRT), which are demonstrated to be transcriptional repressors $[13,16,17]$.

\section{Delta-like ligand 4 expression in tumor tissues}

As mentioned previously, one of the most significant components of the Notch signaling pathway considered as a promising target in angiogenesis- based cancer therapy is DLL4 [1]. The activity and expression pattern of DLL4 suggest a significant role for this ligand in the control of endothelial cell biology $[18,19]$. During embryonal development DLL4 plays a pivotal role in determination and regulation of vasculogenesis. For example, in mDLL4 conditionally overexpressed transgenic mice, double transgenic embryos have shown enlarged dorsal aortae and died before embryonic day 10.5. Probably, this lethal effect was the consequence of premature arteriovenous fusion. It should be mentioned that reduced vascular sprouting, proliferation and migration of endothelial cells were also noted. In vitro and in vivo studies of DLL4-FL (DLL4-full length) expression in endothelial cells recapitulate a great number of DLL4 transgenic finding, e.g. decreased tube formation, reduced vascular branching, increased pericyte recruitment and increased fibronectin expression [20]. It is worth noting that different Notch ligands have shown different places of expression during vascular morphogenesis [21]. For example, Jag1 expression was demonstrated to be present in stalk cells of the leading vasculature and at the arterial branch points. In those sites DLL4 expression was not detected. In contrast, DLL4 was the only ligand exhibited on tip cells at the end of growing vascular sprouts. It was also present in stalk cells, capillaries, arterial endothelium and in mural cells of mature arteries [21].

As revealed by the studies, DLL4 expression in human adult vasculature is low but in the tumor vessels is upregulated [1, 22-24]. For example, the expression of DLL4 within the vasculature of clear-cell renal cell carcinoma was upregulated almost 9-fold more than in normal kidney tissue and was correlated with the level of VEGF. What is interesting, the expression of DLL4 in endothelial cells was just upregulated by VEGF and basic fibroblast growth factor (bFGF) synergistically. It is worth noting that downregulation of DLL4 may have led to inhibition of endothelial cell proliferation, migration and network formation [25]. DLL4 is also associated with vascular differentiation in bladder cancer. The expression of DLL4 was

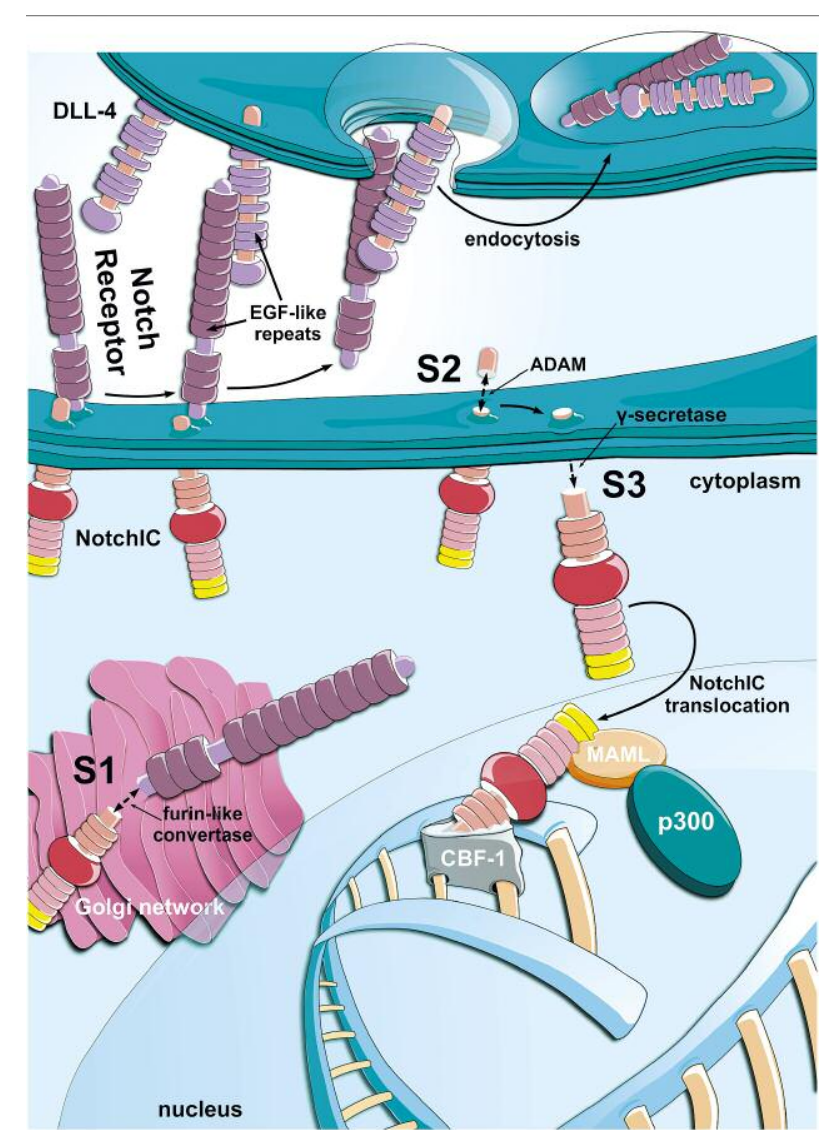

Fig. 1. Notch signaling pathway - details in the text

significantly upregulated in the case of superficial and invasive bladder cancer and was clearly correlated with CD34 and VEGF expression [26]. In clinical breast cancer samples DLL4 was expressed by intratumoral endothelial cells (almost 100\%) and cells of ductal carcinomas. High intensity of DLL4 endothelial expression was a statistically significant adverse prognostic factor, which may suggest that breast tumors with high DLL4 expression in the vasculature progressed more rapidly [27]. In contrast to the case of bladder and breast cancer, endothelial expression of DLL4 in colon cancer should not be considered as a prognostic factor. The expression of DLL4 in colon cancer tissues was observed preferentially in endothelium and, what is not surprising, was significantly associated with expression of VEGF [28]. Moreover, neoplastic cells, especially goblet cells, have also shown expression of DLL4. The detection of DLL4, Notch1 and Notch4 in thyrocytes and their regulation in various thyroid pathologies suggest that Notch signaling may also play a role in thyroid carcinogenesis and angiogenesis. The expression of DLL4 was analyzed in normal thyroids, hyperplastic thyroids from patients with Graves' disease, microcarcinomas and follicular carcinomas. Immunohistochemical expression of DLL4 was highly variable in thyrocytes from normal thyroids and from patients with Graves' disease. In tumors, expression of DLL4 was homogeneous and intense. It is worth emphasizing that in Graves' patients only capillary endothelial cells were positive for DLL4 staining whereas in carcinomas the expression was detected in large vessels [29]. The study of Hu et al. reported that DLL4 plays a pivotal role in ovarian 




Fig. 2. Cancer angiogenesis inhibition by way of VEGF blockade

cancer pathogenesis. It seems that in this case, DLL4 overexpression is an independent predictor of poor survival. Patients with tumors responding to anti-VEGF therapy had lower levels of DLL4 than patients with stable or progressive disease. What is important, under hypoxic conditions VEGF has increased DLL4 expression in the tumor vascular network. Moreover, immobilized DLL4 downregulated VEGFR2 expression in endothelial cells. Probably in this process the mechanism of VEGFR2 promoter methylation was very significant. It should also be noted that combining DLL4-targeted siRNA with bevacizumab resulted in greater inhibition of tumor growth, compared with control or treatment with bevacizumab alone. Therefore, DLL4 targeting in combination with anti-VEGF treatment might improve outcomes of ovarian cancer treatments [30].

\section{Delta-like ligand 4 as a promising target in angiogenesis-based cancer therapy}

In clinical practice, angiogenesis inhibition by way of VEGF blockade is demonstrated to produce a great number of benefits [31, 32] (Fig. 2). Therefore, several approaches have been taken to target angiogenesis in cancer: drugs that target one or more soluble ligands of the VEGF family, drugs that selectively inhibit some of the receptors from the VEGF family and drugs that inhibit VEGF receptors among other non-VEGFrelated targets. It has been reported that two compounds may have significant clinical activity: bevacizumab and aflibercept $[32,33]$. For example, in epithelial ovarian cancer bevacizumab has shown efficacy as monotherapy and combined with chemotherapy in both the first-line and recurrent settings $[34,35]$. Moreover, the use of bevacizumab during up to 10 months after carboplatin and paclitaxel chemotherapy prolongs median progression-free survival (PFS) by about 4 months in patients with advanced epithelial ovarian cancer [36]. Nevertheless, resistance to VEGF inhibitors is emerging as a significant problem of clinical oncology [22]. Studies providing a rational basis for development of novel anti-angiogenic strategies are needed. In this context DLL4 targeting might represent an efficacious approach for the treatment of solid malignancies [37]. As revealed by the studies, blockade of DLL4 resulted in tumor growth inhibition asso- ciated with the formation of a non-functional, hypersprouting tumor vasculature which rendered the tumors more hypoxic. In tumors treated with DLL4 inhibitors reduced pericyte coverage and increased vascular leakage have been reported. An increase of vascular leakiness connected with impaired vascular integrity may explain a rapid decrease in tumor perfusion. These findings clearly provide a rationale for DLL4 targeting for improved treatment of cancer patients [38].

What about the ways of DLL4 targeting? As mentioned above, one of them is specific targeting using anti-DLL4 antibodies. Unlike $\gamma$-secretase inhibitors, anti-DLL4 antibodies did not induce gastrointestinal toxicity. They have no discernible impact on goblet cell differentiation or crypt progenitor cell proliferation. Hoey et al. found that selective antihuman antibody $21 \mathrm{M} 18$ inhibited expression of Notch target genes including HES1 and ATOH1, which in effect caused a low level of malignant cell proliferation [39]. What is important, administration of DLL4 neutralizing antibody was probably responsible for rapid changes in mouse liver gene expression associated with proliferation and cell cycle regulation. Moreover, animals models such as mice, rats and cynomolgus monkeys exposed to anti-DLL4 antibody have developed strong changes in the liver tissue including profound sinusoidal dilation and centrilobular hepatocyte atrophy. It is thought that those changes were the effect of DLL4 inhibition through Notch1 receptor. Why? Because similar changes were detected when Notch1 signaling was suppressed by $\gamma$-secretase or drugs targeting Notch1 receptors [40,41]. The findings of these studies may suggest that DLL4 signaling plays a pivotal role in maintaining liver structural and functional integrity. Apart from liver structural changes observed upon anti-DLL4 treatment other concerning safety signals have been reported following prolonged administration. For example, in male rats, skin lesions with features of vascular neoplasms were observed. Additionally, rare tumors with similar features were indentified in heart and lung [41]. It should be mentioned that proliferative vascular alterations were demonstrated only in rats after 8 weeks of continuous anti-DLL4 administration. The consequence of longer exposure in other species remain unknown [41]. A very important question from the clinical point of view is what type of tumor would benefit most from therapy with the use of anti-DLL4 antibodies?

Over the last years, the concept of using the immune system to fight tumors has been reported [42]. Immunization with DLL4-encoding plasmid DNA by in vivo electroporation retarded the growth of orthotopically implanted mammary carcinomas in mice. Probably the main mechanism involved in this process was connected with targeting endothelial tip cells and DLL4 ligand. Importantly, no evidence for delayed wound healing responses or for toxicity associated with pharmacological DLL4 blocking was reported in mice immunized with the DLL4 vaccine. Induction of immunity toward mediators of pathological angiogenesis such as DLL4 will be a significant step in clinical oncology because it could provide protection against recurrent malignancies in the adjuvant setting [43].

In summary, in recent years DLL4 has appeared as a significant regulator of tumor angiogenesis. Blockade of this ligand results in non-productive angiogenesis with an inhibitory effect on tumor growth. Nevertheless, the careful evaluation 
of adverse effects on physiology of normal organs in relation to therapeutic doses of DLL4 inhibitors will be critical for advancement of DLL4 blocking agents in clinical oncology.

I would like to express my gratitude to M.Sc. M. Michalski for his skilled technical assistance.

The authors declare no conflict of interest.

\section{References}

1. Sainson RC, Harris LC. Anti- Dll4 therapy: can we block tumour growth by increasing angiogenesis? Trends Mol Med 2007; 13: 389-95.

2. Bridges EM, Harris AL. The angiogenic process as a therapeutic target in cancer. Biochem Pharmacol 2011; 15; 81: 1183-91.

3. Rosca EV, Koskimaki JE, Rivera CG, Rivera CG, Pandey NB, Tamiz AP, Popel AS. Anti- angiogenic peptides for cancer therapeutics. Curr Pharm Biotechnol 2011; 12: 1101-16.

4. Goel S, Duda DG, Xu LM, Munn LL, Boucher Y, Fukumura D, Jain RK. Normalization of the vasculature for treatment of cancer and oth er diseases. Physiol Rev 2011; 91: 1071-121.

5. Białas M, Dyduch G, Adamek D. The tumor and its microenvironment - a complicated interpaly. Wspolczesna Onkol 2011; 15: 305-308.

6. Tortora G, Melisi D, Ciardello F. Angiogenesis: a target for cancer therapy. Curr Pharm Dess 2004; 10: 11-26.

7. Biedka M, Makarewicz R Lebioda A, Kardymowicz H, Goralewska A. Naczyniowo-śródbłonkowy czynnik wzrostu C jako czynnik predyk cyjny w raku szyjki macicy? Wspolczesna Onkol 2010; 14: 87-92.

8. Petrillo M, Scambia G, Ferrandina G. Novel targets for VEGF-independent anti- angiogenic drugs. Expert Opin Investig Drugs 2012 21: 451-72.

9. Cao Y. Antiangiogenic cancer therapy. Semin Cancer Biol 2004; 14: 139-45.

10. Shibuya M. Differential roles of vascular endothelial growth factor receptor-1 and receptor-2 in angiogenesis. J Biochem Mol Bio 2006; 39: 469-78.

11. Bishop-Bailey D. Tumour vascularisation: a druggable target. Curr Opin Pharmacol 2009; 96-101.

12. Phng LK, Gerhardt H. Angiogenesis: a team effort coordinated by notch. Dev Cell 2009; 16: 196-208

13. Garcia A, Kandel JJ. Notch: a key regulator of tumor angiogenesis and metastasis. Histol Histopathol 2012; 27: 151-6.

14. Miyamoto S, Rosenberg DW. Role of Notch signaling in colon homeostasis and carcinogenesis. Cancer Sci 2011; 102: 1938-42.

15. Lobov IB, Renard RA, Papadopoulous N, Gale NW, Thurston G, Yan copoulos GD, Wiegand SJ. Delta- like ligand 4(D\|4) is induced by VEGF as a negative regulator of angiogenic sprouting. Proc Natl Acad Sci U S A 2007; 104: 3219-24.

16. Guo S, Liu M, Gonzalez-Perez RR. Role of Notch and its oncogenic signaling crosstalk in breast cancer. Bichim Biophys Acta 2011; 1815: 197-213.

17. Izrailit J, Reedijk M. Developmental pathways in breast cancer and breast tumor- initiating cells: therapeutics implications. Cancer Lett 2012; 317: 115-26.

18. Shutter JR, Scully S, Fan W, Richards WG, Kitajewski J, Deblandre GA, Kintner CR, Stark KL. DLL4, a novel Notch ligand expressed in arterial endothelium. Genes Dev 2000; 14: 1313-8

19. Duarte A, Hirashima M, Benedito R, et al. Dosage-sensitive requirement for mouse Dll4 in artery development. Genes Dev 2004; 18: 2474-8.

20. Trindade A, Kumar SR, Scehnet JS, et al. Overexpression of delta-like 4 induces arterialization and attenuates vessel formation in developing mouse embryos. Blood 2008; 1125: 1720-9.

21. Hofmann JJ, Luisa Iruela- Arispe M. Notch expression patterns in the retina: An eye on receptor-ligand distribution during angiogenesis. Gene Expr Patterns 2007; 7: 461-70.

22. Li JL, Sainson RC, Shi W, et al. Delta-like 4 Notch ligand regulates tumor angiogenesis, improves tumor vascular function, and promotes tumo growth in vivo. Cancer Res 2007; 1; 67: 11244: 53.
23. Segarra M, Williams CK, Sierra Mde L, et al. Dll4 activation of Notch signaling reduces tumor vascularity and inhibits tumor growth. Blood 2008; 112: 1904-11.

24. Li J, Sainson RC, Oon CE, et al. DLL4-Notch signaling mediates tumor resistance to anti-VEGF therapy in vivo. Cancer Res 2011; 71: 6073-83.

25. Patel NS, Li JL, Generali D, Poulsom R, Cranston DW, Harris AL. Upregulation of delta-like 4 ligand in human tumor vasculature and the role of basal expression in endothelial cell function. Cancer Res 2005; 65: 8690-7.

26. Patel NS, Dobbie MS, Rochester M, et al. Up-regulation of endothelial delta-like 4 expression correlates with vessel maturation in bladder cancer. Clin Cancer Res 2006; 12: 4836-44

27. Jubb AM, Soilleux EJ, Turley H, et al. Expresion of vascular notch ligand delta-like 4 and inflammatory markers in breast cancer. Am J Pathol 2010; 176: 2019-28.

28. Jubb AM, Turley H, Moeller HC, et al. Expression of delta-like ligand 4 (DIl4) and markers of hypoxia in colon cancer. Br J Cancer 2009; 101: 1749-57.

29. Geers C, Colin IM, Gerard AC. Delta- like 4/Notch pathway is differentially regulated in benign and malignant thyroid tissues. Thyroid 2011; 21: 1323-30.

30. Hu W, Lu C, Dong HH, et al. Biological roles of the Delta family Notch ligand Dll4 in tumor end endothelial cells in ovarian cancer. Cancer Res 2011; 71: 6030-9.

31. Verheul HM, Pinedo HM. Possible molecular mechanisms involved in the toxicity of angiogenesis inhibition. Nat Rev Cancer 2007; 7 : 475-85.

32. Bagnasco L, Piras D, Parodi S, Bauer I, Zoppoli G, Patrone F, Ballestrero A. Role of angiogenesis inhibitors in colorectal cancer: sensitive and insensitive tumors. Curr Cancer Drug Targets 2012; 12: 303-15.

33. Takahashi S. Vascular endothelial growth factor (VEGF), VEGF receptors and their inhibitors for antiangiogenic tumor therapy. Bioll Pharm Bull 2011; 34: 1785-8.

34. Teoh D, Secord AA. Antiangiogenic agents in combinations with chemotherapy for the treatment of epithelial ovarian cancer. In J Gynecol Cancer 2012; 22: 348-59.

35. Matulonis UA. Bevacizumab and its use in epithelial ovarian cancer. Future Oncol 2011; 7: 365-79.

36. Burger RA, Brady MF, Bookman MA, et al. Incorporation of bevacizumab in the primary treatment of ovarian cancer. N Engl J Med 2011; 365: 2473-83.

37. Ridgway J, Zhang G, Wu Y, et al. Inhibition of Dll4 signalling inhibits tumour growth by deregulating angiogenesis. Nature 2006; 444: 1083-7.

38. Kuchnert F, Kirschner J.R, Thurston G. Dll4-Notch signaling as a therapeutic target in tumor angiogenesis. Vasc Cell 2011; 3: 20.

39. Hoey T, Yen WC, Axelrod F, et al. DLL4 blockade inhibits tumor growth and reduces tumor-initiating cell frequency. Cell Stem Cell 2009; 5: 168-77.

40. Yan M, Callahan CA, Beyer JC, Allamneni KP, Zhang G, Ridgway JB, Niessen K, Plowman GD. Chronic DLL4 blockade induces vascular neoplasms. Nature 2010; 463: E6-7.

41. Yan M. Therapeutic promise and challenges of targeting DLL4/NOTCH1. Vasc Cell 2011; 8: 3-17.

42. Pejawar-Gaddy S, Finn OJ. Cancer vaccines: accomplishments and challenges. Crit Rev Oncol Hematol 2008; 67: 93-102.

43. Haller BK, Brlve A, Wallgard E, et al. Therapeutic efficacy of a DNA vaccine targeting the endothelial tip cell antigen delta-like ligand 4 in mammary carcinoma. Oncogene 2010; 29: 4276-86.

\section{Address for correspondence}

\section{Marlena Brzozowa}

Chair and Department of Histology and Embryology

Medical University of Silesia

Jordana 19

41-808 Zabrze, Poland

e-mail: marlena.brzozowa@op.pl

Submitted: $\quad 31.05 .2012$

Accepted: $\quad 16.08 .2012$ 